\title{
Decision support in the information maintenance of individual education trajectory based on ontological models and distributed RDF-storage
}

\author{
A Klimova ${ }^{1}$, N Yusupova ${ }^{1}$, O Smetanina ${ }^{1}$, A Kovtunenko ${ }^{1}$ \\ ${ }^{1}$ Ufa State Aviation Technical University, 12 Karl Marx str., Ufa, Russia
}

\begin{abstract}
The article considers the problem of decision support in the information maintenance of individual education trajectory. The development of algorithms and software for processes automating in educational systems requires a special approach. The use of ontological models makes it possible to unify the description of the elements of educational systems and apply the technology of digital footprints when managing business processes in educational systems. An ontological model for managing educational trajectories is proposed. An algorithm has also been developed to compare competence-based models of curricula using latent-semantic analysis. The proposed models and methods are implemented as a distributed software system using the semantic web approach on the basis of distributed RDF-storage. The effectiveness of the developed algorithms and software solutions is demonstrated by the example of academic mobility in Ufa State Aviation Technical University. Prospects for further research in this area are outlined.
\end{abstract}

\section{Introduction}

The effectiveness of functioning for an organization or its structural unit depends, to a large extent, on many factors, including the quality of decisions. In turn, decision makers now often must work with heterogeneous data. The limitations of existing methods and models of decision making lead to a contradiction. On the one hand, processing of heterogeneous data is necessary for making a decision, and on the other hand, existing methods and means for processing such data and obtaining conclusions on them are not fully developed for a number of tasks and domains. One of the ways to overcome this contradiction in modern conditions is the development of decision support systems based on ontological engineering.

Ontologies are developed and can be used to solve tasks in various fields of knowledge (including education), namely, where data processing that takes into ac-count their semantics is required.

Functionally, ontologies can be used for sharing by people / agents (software), ac-cumulating and reusing knowledge in applications, creating models and programs that use ontologies [1]. Ontologies can be used to describe learning material that compose a course, capable of providing adaptive elearning environments and reusable educational resources [2], for user's knowledge requirement acquisition and analysis [3].

In applications related to educational activities, developed ontologies are used to solve tasks of data search [4], information and knowledge exchange [5], knowledge management [6], semantics of heterogeneous resources [7], etc. 
Many works using ontologies in such applications are devoted to e-learning [7], in particular, ELSE system [8], OLOUD system [9], social e-learning system TagSES [10], Semantic Web System [11] formation of educational programs for the training of students [12], automated development of educational ontologies of knowledge areas [13], construction of educational trajectories, taking into account various factors [14], etc.

This article contains the analysis of ontologies in applications for educational systems and discusses issues of creation and use of ontologies for data search, formation of new knowledge, formulation of the task for the development and use of ontology in an application that provides the construction of an individual education-al trajectory.

\section{State of the issues of creating and using ontologies in education}

Ontological engineering involves creating ontologies while taking into account the use of objectoriented and structural analysis.

From the subject-problem knowledge according to [15] in the general case, concepts and processes of the subject domain, concepts and classes of tasks, as well as methods for solving the latter are distinguished. The algorithms that implement corresponding methods are also included here. The listed types of knowledge are divided into static and dynamic. By the ontological scheme they are divided into: ontology of objects ("thesaurus"); ontology of processes, ontology of applications, ontology of the class of tasks and ontology of methods for solving problems.

All concepts [15] are divided into many classes: depending on the display of the species or kind of objects - on species and generic concepts; depending on the display of a part or a whole of objects - on the concepts of part and concept-whole, depending on the number of displayed objects - on single and general concepts; depending on the display of the object or property abstracted from the subject specific concepts and abstract ones.

The most common knowledge representation languages used to describe ontologies included in various applications, are RDF, its semantic extension of RDFS and OWL. The main element of RDF is the triad of subject, predicate and object. A predicate is a binary relation between a subject and an object. OWL-ontologies can contain descriptions of classes, properties, and their instances. OWL is characterized by two categories of properties: property-objects and property-values. Instances (individuals) are determined by using the axioms of instances. The most famous ontology editors are Protégé, Ontomaster, WordNet, and others.

A detailed survey of currently known researches in the field of creation and use of ontologies in education [16] was conducted by Volegzhanina I.S. The author shows the place of ontological research in modern Russian education and the actual directions of further research. Noted: conceptual level (ontologies in the education system (distance learning, mobile learning), their didactic, cognitive and social functions, se-mantic models of competences) and practical level (creating ontologies of domains, including ontological glossaries, creating information portals for users' work with ontologies, ontologies as a diagnostic tool). Many publications of foreign researchers also contain the results of using the ontological approach in educational processes. Most of them consider the solution of the problem of electronic education.

Among the specialists engaged in ontological engineering in the field of education, now it is possible to note Chen B., Gascueña J. M., Kim H., Lo W.-Sh., Mizoguchi R., Sabarish R., Zeng Q., etc.

Thus, the analysis of the developed ontological models allowed to distinguish both general classes regardless of the tasks to be solved (normative documents, discipline, competence, etc.) and classes directly related to the purpose of the application - soft-ware (hardware, software), literature sources, educational process, etc.

The authors of the article added classes related to the content of the task to a number of general classes when creating an ontology for an application that provides the formation of an individual educational trajectory of students, oriented only to the content part (mastered competences, their connection with disciplines as a result of their mastering) (see section 4). 
The authors have been engaged for years in the tasks of comparative analysis of educational programs for the training of informatics by Russian and foreign universities before solving the task of forming an individual educational trajectory for student from the point of view of a competence approach. The purpose of the research was to study the experience of leading universities in order to form competitive curricula, including individual ones (taking into account the content aspect of disciplines, the professional needs of the, labor market requirements $[12,17])$.

\section{Statement of the problem}

The process of planning an individual educational trajectory is associated with processing of heterogeneous data. When planning it, documents containing a list of disciplines are used (for example, academic reference, curriculum). The result of this planning is also a document that includes a list of disciplines (for example, the minutes of the examination board meeting, or an individual curriculum). However, result-oriented mastered competences are revealed. Therefore, when planning an individual educational trajectory, competences are identified, both mastered at this stage and planned for mastering. Thus, one of the subtasks in planning the individual educational trajectory is the formalization of the expert's knowledge about the correlation of competences and disciplines. Accounting for the educational standards created by various groups of developers (for different direction of training), competences will have various descriptions, even with their semantic similarity. To unify the presentation of information about the educational process, the following ontological model is proposed.

\subsection{Ontological model of domain}

Ontological model of educational system includes the following elements:

- STRING, INTEGER are the simple types, strings and natural numbers respectively,

- $\quad$ STUD is the set of students,

- $C$ is the set of curricula,

- $D$ is the set of disciplines,

- ACTIVITIES is the set of education activities, provided by university (lectures, practices, lab classes, seminars, traineeship, excursions, etc),

- REFERENCES is the set of references for independent discipline studying (books, internet hyperlinks),

- $\Omega$ is the set of competences,

- $T E R M S \subset I N T E G E R$ is the set of term numbers,

- $Y E A R S \subset I N T E G E R$ is the set of study years numbers.

The main document that gives the right to educational activities within some direction is the main professional education program (MPEP). It is based on curriculum and it can be characterized by the following relations.

- Head :C $\rightarrow$ STRINGS, Head(c) - the heading of direction of the curriculum $c$,

- Cont : $C \rightarrow 2^{D}$, Cont $(c) \subseteq D$ - the subset of disciplines that make up the curriculum $c$.

Each discipline is characterizing by the following relations.

- Title: $D \rightarrow$ STRING, Title (d) - the title of the discipline,

- Comp : $D \rightarrow 2^{\Omega}, \operatorname{Comp}(d) \subseteq \Omega$ - a subset of competences that are mastered in the study of the discipline,

- Work :D $\rightarrow$ INTEGER, Work $(d)$ - workload of the discipline in academic hours,

- Sem: $D \rightarrow$ SEMESTERS, Sem $(d)$ - the number of terms when the intermediate of control of the discipline is held

- $E: D \rightarrow 2^{\text {ACTIVITIES }}$ - the subset of educational activities in discipline study,

- $R: D \rightarrow 2^{\text {REFERENCES }}$ - the subset of auxiliary materials and sources for the discipline study. 
Each student is characterized by the following relations.

- Name $: S T U D \rightarrow$ STRING, Name(stud) - a student's name,

- Plan: STUD $\rightarrow$ C, Plan(stud) - the curriculum according to which a student studies.

\subsection{Formulation of the individual educational trajectory management problem}

Individual educational trajectory is a sequence of educational activities sorted by the year of study. It can be defined as a relation Trajectory:STUD $\times Y E A R S \rightarrow 2^{\text {ACIVITIES }}$. Thus, Trajectory (stud, $y$ ) $\subseteq$ ACTIVITIES is the subset of educational activities for a student held on the $y$-th year of study. Without an individualization it is possible to talk about ideal educational trajectory, which is fully defined by the curriculum of study direction.

$$
\text { Trajectory } *(e p, y)=\underset{\left.d \in \operatorname{Sem}^{-1}(2 y-1) \cap \operatorname{Cont}(e p)\right)}{\bigcup} \in(d) \underset{\left.d \in \operatorname{Sem}^{-1}(2 y) \cap \operatorname{Cont}(e p)\right)}{\bigcup}
$$

At each step of the educational trajectory, a student has current achievements, which are fixed by intermediate control.

$$
\text { CurAchive : STUD } \times Y E A R S \rightarrow 2^{D}, \text { CurAchive }(\text { stud }, y) \subseteq(\text { Cont } \circ \text { Plan })(\text { stud })
$$

An academic debt for the stud on y-th year of study can be defined as follows.

CurDebt $($ stud,$y)=\bigcup_{k=1}^{2(y-1)}\left[\operatorname{Sem}^{-1}(k) \cap(\right.$ Cont $\circ$ Plan $)($ stud $) \backslash$ CurAchive $($ stud,$\left.y-1)\right]$

Thus, management of individual educational trajectory consists of the following steps:

- $\quad$ fixing study achievements,

- $\quad$ making the list of debts,

- $\quad$ making a decision about admitting a student to the next stage (the next year of study) or sending him or her down,

- $\quad$ making the list of auxiliary materials for the debt elimination.

The decision about a student's admission to the next stage is regulated by the inner normative documents of a university. As an example, the admission can be performed if the total discipline workload of debts does not exceed the predefined limit.

$$
\sum_{d \in \text { CurDept }(\text { stud }, y)} \operatorname{Work}(d) \leq W^{*}
$$

here $W^{*}$ - maximum allowed value of total workload.

Thus, solving the problem of management of individual educational is trivial, because it is fully defined by the curriculum of training direction.

Individual educational trajectory can be considered like that at least two curricula (basic and receiving) take part in the student's education. The main problem in this case is the adoption by the second curriculum of educational results which were achieved studying with the first one.

Let $s t u d_{0}$ is a student, who realizes his or her right to individual educational trajectory. He or she finishes $k$-th study year with curriculum $c_{0}\left(\operatorname{Plan}\left(\operatorname{stud}_{0}\right)=c_{0}\right)$ and is transferred to another curriculum as a student $\operatorname{stud}_{1}\left(\operatorname{Plan}\left(\operatorname{stud} d_{1}\right)=c_{1}\right)$. And let $D^{\text {basic }}=\operatorname{CurAchive}\left(\operatorname{stud}_{0}, k\right)$ be a set of disciplines studied according to the basic curriculum.

To form a new item of individual educational trajectory after transferring it is necessary to estimate a student's achievements reached before in the terms of the second curriculum. It means that we need to make a list of disciplines, that will be considered as studied ones. To do this we need to establish the matching between disciplines of basic and receiving curriculum. 
In fact, the individual educational trajectory contains the disciplines both of basic and receiving curricula. But formally we are unable to manage the educational process, because a student hasn't studied any disciplines on receiving direction. Due to this, the information maintenance of the individual educational trajectory includes the making of the following interrelated decisions.

- $\quad$ Making a list of disciplines $D^{*}$ of the receiving curriculum, that will be considered as the studied ones.

- $\quad$ Finding out the number of study year for a student's admission.

$$
\begin{gathered}
\text { CurDebt }\left(\text { stud }_{1}, y\right)=\bigcup_{i=1}^{2(y-1)}\left[\text { Trajectory }^{*}\left(c_{1}, i\right)\right] \backslash D * \\
m=\arg \min _{y \in Y E A R S}\left|\operatorname{CurDebt}\left(\operatorname{stud}_{1}, y\right)\right| \\
\text { CurAchive }\left(\operatorname{stud}_{1}, m\right)=D^{*}
\end{gathered}
$$

Making a list of the disciplines, that must be studied on the selected stage (study year) according to curriculum (an academic debt). During the first year on new direction a student must pass an intermediate control on them independently.

Making an individual educational trajectory with considering the "passed" disciplines.

$$
\operatorname{Trajectory}\left(\operatorname{stud}_{1}, m\right)=\bigcup_{d \in \operatorname{CurDebt}\left(\operatorname{stud}_{1}, m\right)} E(d)
$$

To compare the disciplines, it is offered to use the semantic analysis of mastered competences.

Let us introduce a measure of the difference between two competences as a real-valued function of two arguments, which takes values from a range from 0 (absolutely different competences) to 1 (literally matching competences). Also we introduce the function of the effectiveness of the "passed" list as the least degree of similarity between the closest competences mastered by a student in the basic direction and the competences of the receiving direction.

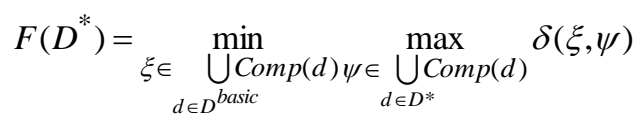

The problem of the individual educational trajectory formation is to find out the best "passed" list.

$$
D_{\text {best }}=\arg \max _{D^{*} \in 2^{\text {Cont }\left(c_{1}\right)}} F\left(D^{*}\right)
$$

In case when the transfer is carried out between different directions (or with international traineeship) the task of comparing competence models of two curricula is especially acute. This is due to the fact that the competences of the directions involved in the individual educational trajectory are similar either partially (if the directions belong to the same group) or do not completely coincide (if the directions either belong to different groups, or in case of international traineeship).

\section{Competence models matching using latent semantic analysis}

The main procedure in solving the problem of matching competence models is a pairwise estimation of the degree of semantic similarity of the competences of the basic and the receiving curricula.

Competence is a small text describing any completed labor function that a graduate is able to perform. Let each competence $\omega$ be considered as a multiset generated by the set of all linguistic units $V$ used in the source texts of competences. According to the definition, a multiset is an ordered pair $\omega=\left\langle V, m_{\omega}\right\rangle$, where: $V-$ is the generating set (domain), $m_{\omega}(v)-$ is the multiplicity function of the multiset, indicating how many times each element of the generating set $V$ is included into the multiset $\omega$. 
To solve the problem in the article it is offered to use the following algorithm.

1. As part of the texts pre-processing, it is proposed to produce:

- exclusion of stop words;

- lemmatization;

- tokenization using N-grams.

2. For vectorization, it was decided to use the TF-IDF method, according to which the components of the vector representation of competence represent the ratio of the frequency of occurrence of a given word to the total number of words in the competence. For each term $\mathrm{v}$ and each competence $\omega$, this number is determined by the relation

$$
\operatorname{tfidf}(v, \omega, \Omega)=\frac{m_{\omega}(v)}{|\omega|} \ln \frac{|\Omega|}{\sum_{\omega \in \Omega} \chi_{\omega}(v)},
$$

where $\chi_{\omega}(v)=\left\{\begin{array}{l}1, v \in \omega \\ 0, v \notin \omega\end{array}\right.$ is a characteristic function of the multiset $\omega,|\Omega|$ is the total number of competences considered.

Thus, the vector representation of the competence $\omega$ :

$$
\mathbf{X}(\omega)=\left(\begin{array}{c}
\operatorname{tfidf}\left(v_{1}, \omega, \Omega\right) \\
\vdots \\
\operatorname{tfidf}\left(v_{|V|}, \omega, \Omega\right)
\end{array}\right),
$$

where $v_{i} \in V$ are the terms included in the corpus $V$.

3. It is proposed to carry out semantic factorization of texts by the method of constructing a singular decomposition. For this, a matrix of vectorized texts is formed.

$$
\mathbf{M}=\left(\begin{array}{ccc}
\operatorname{tfidf}\left(v_{1}, \omega^{1}, \Omega\right) & \cdots & \operatorname{tfidf}\left(v_{1}, \omega^{|\Omega|}, \Omega\right) \\
\vdots & \ddots & \vdots \\
\operatorname{tfidf}\left(v_{|V|}, \omega^{1}, \Omega\right) & \cdots & \operatorname{tfidf}\left(v_{|V|}, \omega^{|\Omega|}, \Omega\right)
\end{array}\right)
$$

The result of the singular decomposition of the matrix $\mathbf{M}$ are the matrices $\mathbf{U}$ and $\mathbf{W}$, and also the diagonal matrix of importance $\boldsymbol{\Lambda}$, the elements of which indicate the degree of importance of one or another semantic factor in the texts of the corpus.

$$
\mathbf{M}=\mathbf{U} \cdot \boldsymbol{\Lambda} \cdot \mathbf{W}^{\mathrm{T}}
$$

The least significant factors can be discarded by zeroing out those elements of the matrix $\boldsymbol{A}$ that are less than a certain value (determined by an expert) to obtain the matrix $\Lambda^{*}$. Based on it, you can get an approximate matrix $\boldsymbol{M}^{*}$ of vector representations of competences in which the least significant semantic factors are not taken into account.

$$
\mathbf{M}^{*}=\mathbf{U} \cdot \boldsymbol{\Lambda}^{*} \cdot \mathbf{W}^{\mathrm{T}}
$$

As a result of the semantic factorization, refined vector representations of competences are obtained.

4. To evaluate the similarity of the two competences, it is proposed to use the cosine metric. Thus, the degree of semantic affinity of two competences will be 


$$
\delta\left(\omega^{1}, \omega^{2}\right)=\frac{\mathbf{X}^{*}\left(\omega^{1}\right)^{\mathrm{T}} \mathbf{X}^{*}\left(\omega^{2}\right)}{\sqrt{\mathbf{X}^{*}\left(\omega^{1}\right)^{\mathrm{T}} \mathbf{X}^{*}\left(\omega^{1}\right)} \sqrt{\mathbf{X}^{*}\left(\omega^{2}\right)^{\mathrm{T}} \mathbf{X}^{*}\left(\omega^{2}\right)}} .
$$

The result of comparing competence models is a matrix, the $(i, j)$-th element of which contains a numerical estimate of the similarity of the $i$-th competence of the basic direction with the $j$-th competence of the receiving direction.

\section{Distributed software architecture based on RDF data representation}

Ontology can also be used in the application which provides the planning of individual educational trajectory within international traineeship (the authors of the article have an extensive base for educational programs of foreign universities [12].

The structural scheme of the developed application (figure 1) includes databases and knowledge base, precedents, models that allow using the developed methods (method of expert assessments, nearest neighbor method) and algorithms (generalized algorithm for solution of integrated task, algorithm of correlation between competences and disciplines with the subsequent choice of rational "passed" list of disciplines) to solve the subtasks of formation of the individual educational trajectory.

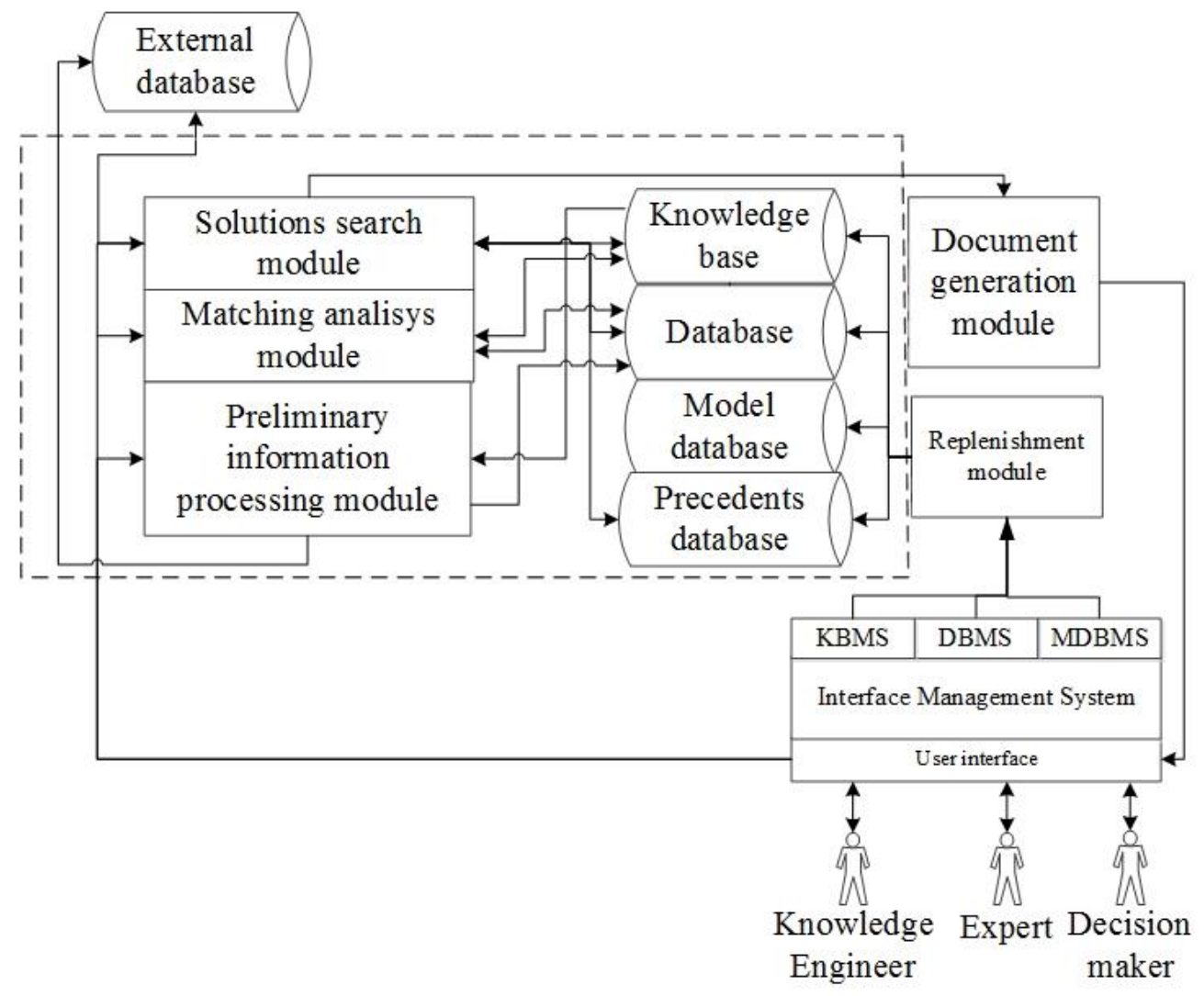

Figure 1. Structural scheme of the application for implementation of the individual educational trajectory management.

The following software architecture is proposed (figure 2). It includes semantic web, which in it's order contains distributed RDF storage. Each part of the RDF storage is deployed in different organization - participant of educational process. 


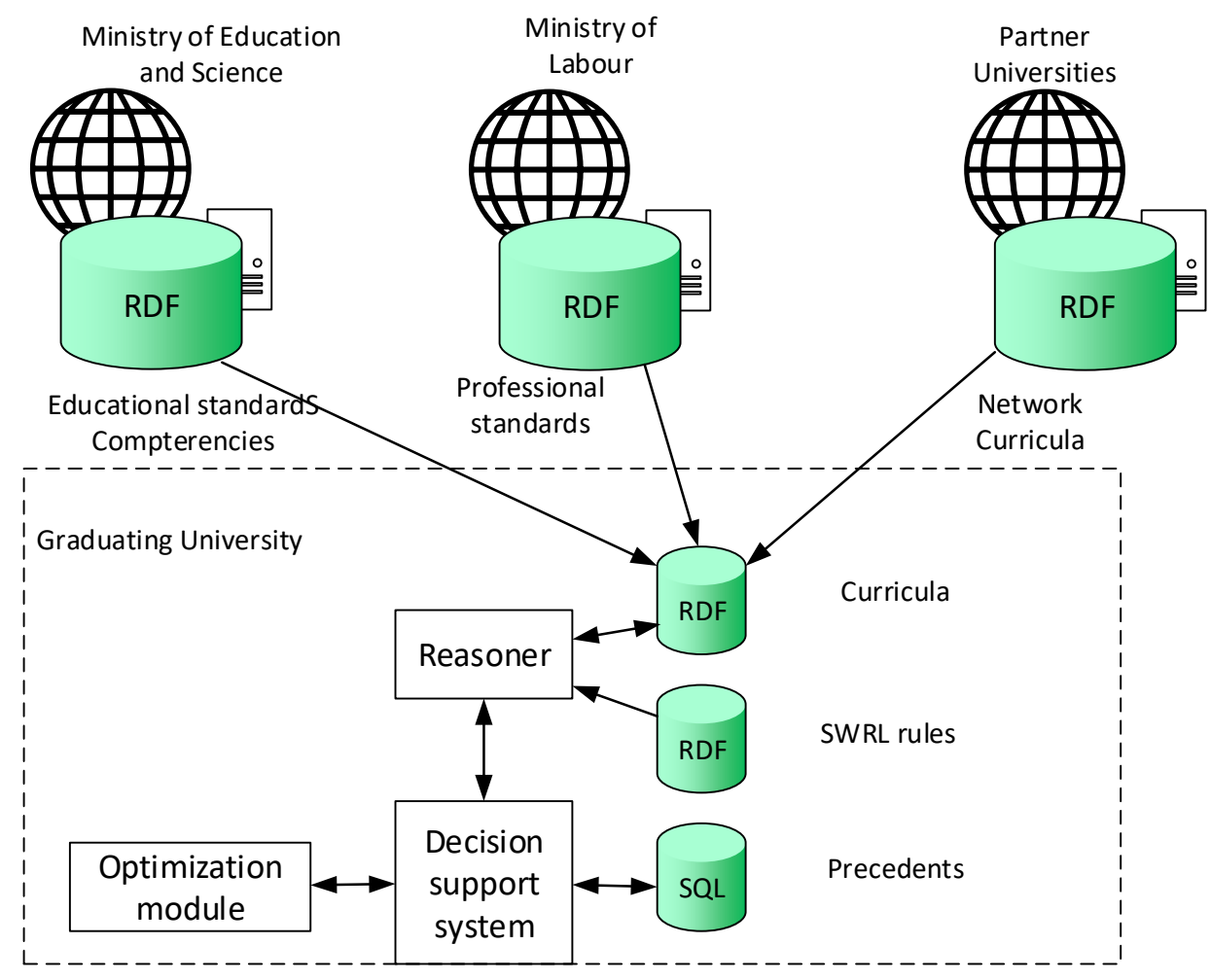

Figure 2. Distributed software system architecture.

Ministry of Education and Science stores the information about educational standards. Ministry of Labor stores everything about professional standards. On the resources of different universities, which are taking a part in network education or traineeship there are placed RDF-storages of their curricula.

The graduating university keeps it's own part of distributed storage. It includes curricula mainly. The mechanisms of digital maintenance contains of the SWRL-rules base, reasoner to keep digital footprints of individual educational trajectory actual, decision support system with GUI, to interact with staff, and optimization module to auto formation variants of effective "passed" lists. Also there is the SQL-base of precedents, that stores a digital footprint of previous decisions.

\section{Experimental verification of developed algorithms}

The developed algorithms were implemented as a module for comparing competence models to support the educational process. Efficiency of the module was evaluated based on the following example. A student of the direction 38.03.05 "Business Informatics" has studied for 2 terms, passed all the exams, and he or she is transferred to the direction 09.03.04 "Software Engineering".

The competences (table 1) that the student mastered in the direction 38.03.05 "Business Informatics" during the year (table 2) were compared with the competence model of the direction 09.03.04 "Software Engineering" (the fragment of which is presented in table 3) to identify competences that are identical and similar in meaning.

Table 1. Notation table.

\begin{tabular}{cc}
\hline Abbreviation & Expansion \\
\hline OC & Overall cultural competences \\
\hline OPC & Overall professional competences \\
\hline PC & Professional competences \\
\hline PSC & Professional specialized competences \\
\hline
\end{tabular}


Table 2. The fragment of the competence model of curriculum "Business Informatics".

\begin{tabular}{|c|c|}
\hline Cypher & Description \\
\hline OC-1 & Ability to use the basics of philosophical knowledge to form a worldview position \\
\hline OC-3 & Ability to use the basics of economic knowledge in various fields \\
\hline OC-4 & Ability to use the basics of legal knowledge in various fields \\
\hline OC-9 & $\begin{array}{c}\text { The ability to use first-aid techniques, methods of protection in emergency } \\
\text { situations }\end{array}$ \\
\hline OPC-1 & $\begin{array}{l}\text { Ability to solve standard tasks of professional activity based on information and } \\
\text { bibliographic culture using information and communication technologies and } \\
\text { taking into account the basic requirements of information security }\end{array}$ \\
\hline OPC-3 & $\begin{array}{l}\text { Ability to work with a computer as a means of information management, work } \\
\text { with information from various sources, including global computer networks }\end{array}$ \\
\hline PC-17 & $\begin{array}{l}\text { Ability to use the basic methods of natural sciences in professional activities for } \\
\text { theoretical and experimental research }\end{array}$ \\
\hline PC-18 & $\begin{array}{l}\text { Ability to use the appropriate mathematical apparatus and tools for processing, } \\
\text { analysis and systematization of information on the research topic }\end{array}$ \\
\hline \multicolumn{2}{|c|}{ Table 3. The fragment of the competence model of curriculum 04 "Software Engineering". } \\
\hline Cypher & Description \\
\hline OC-1 & Ability to use the basics of philosophical knowledge to form a worldview position \\
\hline OC-3 & Ability to use the basics of economic knowledge in various spheres of life \\
\hline OC-4 & Ability to use the basics of legal knowledge in various spheres of life \\
\hline OC-9 & Ability to use first-aid techniques, methods of protection in emergency situations \\
\hline OC-4 & $\begin{array}{c}\text { Knowledge of concepts and attributes of software quality (reliability, security, } \\
\text { usability), including the role of people, processes, methods, tools and technologies } \\
\text { for quality assurance }\end{array}$ \\
\hline PSC-1 & $\begin{array}{l}\text { Ability to apply methods of analysis of the applied field at the conceptual, logical, } \\
\text { mathematical and algorithmic levels }\end{array}$ \\
\hline
\end{tabular}

All competences were tokenized by $\mathrm{N}$-grams method with $\mathrm{N}=4$.

The matrix $\boldsymbol{\Lambda}$ obtained as a result of the singular decomposition was contrasted, that is, the 25 largest elements were saved, and the rest were replaced by 0 to obtain the matrix $\Lambda^{*}$. The matrix $\mathbf{M}^{*}$ obtained after the reconstruction was used to assess the similarity of the competences of basic and receiving directions based on the cosine metric. The results of the algorithm are presented in table 4 .

Table 4. The matrix of pairwise similarity of competences.

\begin{tabular}{ccccccccc}
\hline$\delta\left(\omega^{1}, \omega^{2}\right)$ & OC-1 & OC-3 & OC-4 & OC-9 & OPC-1 & OPC-3 & PC-17 & PC-18 \\
\hline OC-1 & 1 & 0.471 & 0.471 & 0.211 & 0.071 & 0.073 & 0.192 & 0.172 \\
\hline OC-3 & 0.471 & 0.875 & 0.75 & 0.224 & 0.075 & 0.154 & 0.102 & 0.091 \\
\hline OC-4 & 0.471 & 0.75 & 0.875 & 0.224 & 0.075 & 0.154 & 0.102 & 0.091 \\
\hline OC-9 & 0.211 & 0.224 & 0.224 & 1 & 0.067 & 0.069 & 0.183 & 0.082 \\
\hline
\end{tabular}




\begin{tabular}{llllllllc}
\hline PC-4 & 0 & 0 & 0 & 0 & 0.087 & 0.089 & 0 & 0 \\
\hline PSK-1 & 0 & 0 & 0 & 0.091 & 0.062 & 0 & 0.167 & 0.149 \\
\hline
\end{tabular}

Disciplines passed in the basic direction with their competences: Philosophy (OC-1), Microeconomics (OC-3), Law (OC-4), Sociology (OC-3), Linear Algebra (OPC-1, PC-18), Mathematical Analysis (OPC-1, PC-17), Theoretical Foundations of Informatics (OPC-3), Life Safety (OC-9).

The disciplines of the receiving direction, the competences of which are similar to the competences of the basic direction: $\mathrm{D}_{1}$ : Philosophy (OC-1), $\mathrm{D}_{2}$ : Jurisprudence OC-4), $\mathrm{D}_{3}$ : Life Safety (OC-9), $\mathrm{D}_{4}$ : Economics (OC-3), $\mathrm{D}_{5}$ : Probability Theory and Mathematical Statistics (OC-1, PSC-1), $\mathrm{D}_{6}$ : Intellectual Property in IT (OC-4, PC-4), D

Two alternative "passed" lists have been formed by an expert. The problem of automatic creating an effective "passed" list is beyond the scope of this article and can be solved using various discrete optimization methods, for example, using genetic algorithms.

$$
D^{*}{ }_{1}=\left\{D_{1}, D_{2}, D_{3}, D_{4},\right\}, D^{*}{ }_{2}=\left\{D_{1}, D_{2}, D_{3}, D_{4}, D_{5}, D_{6}, D_{7}\right\}
$$

According to the proposed function the effectiveness (9) of each "passed" list is

$F\left(D *_{1}\right)=0.875, F\left(D *_{2}\right)=0.089$

$D_{\text {best }}=\arg \max \left\{F\left(D_{1}{ }_{1}\right), F\left(D^{*}{ }_{2}\right)\right\}=D^{*}{ }_{1}=\left\{D_{1}, D_{2}, D_{3}, D_{4}\right\}$

In this example, among the given variants of a "passed" list, the most effective from the point of view of semantic similarity to the studied disciplines is the first one.

\section{Conclusions}

The results of the application analysis in education, which basically contain ontological models, showed their extensive use in the field of electronic and distance learning, in the search for educational resources, in optimizing the educational process and educational programs, in creating individual educational trajectories.

The technology of ontological modeling and ontological engineering of domain has made it possible to formalize business processes associated with a student's individual educational trajectory, to unify the presentation of heterogeneous information and to develop algorithms and software for decision support in managing the individual educational trajectory.

The developed technology for comparing curricula based on a latent-semantic analysis of competency models makes it possible to take into account all student's academic achievements, even if they obtained outside of the main curriculum. This allows to set arbitrary optimization tasks in the management of educational systems and solve them using modern intelligent technologies.

The practical significance of the proposed methods and algorithms is not limited to the competency-based approach and can be extended to any educational model.

\section{Acknowledgments}

This work was supported by RFBR grant 19-07-00709 A and partially completed as part of a state assignment no. FEUE-2020-0007.

\section{References}

[1] Mizoguchi R, Kasai T, Yamaguchi H and Nagano K 2005 A Semantic Web System for Helping Teachers Plan Lessons Using Ontology Alignment Applications of Semantic Web Technologies for E-Learning pp 9-18

[2] Gascueña J M, Fernández-Caballero A and González P 2006 Domain Ontology for Personalized E-Learning in Educational Systems 6th IEEE International Conferenceon Advanced Learning Technologies pp 456-458

[3] Zeng Q, Zhao Z and Liang Yo 2010 Course Ontology-based User's Knowledge Requirement Acquisition from Behaviors within E-Learning Systems Bulletin of advanced technology research vol 4 chapter 4 pp 21-29 
[4] Tarus J K, Niu Z and Yousif A 2017 A hybrid knowledge-based recommender system for elearning based on ontology and sequential pattern mining Future Generation Computer Systems vol 72 pp 37-48

[5] Machova K, Vrana J, Mach M and Sincak P 2016 Ontology Evaluation Based on the Visualization Methods, Context and Summaries Acta Polytechnica Hungarica vol 13 chapter 4 pp 53-76

[6] Barao A, Vasconcelos J B, Rocha A and Pereira R 2017 A knowledge management approach to capture organizational learning networks International Journal of Information Management vol 37 chapter 6 pp 735-740

[7] Oprea M 2016 A case study of collaborative ontology development for higher education International Journal of Artificial Intelligence vol 14 chapter 2 pp 70-97

[8] Barbagallo A and Formica A 2017 ELSE: an ontology-based system integrating semantic search and e-learning technologies Interactive Learning Environments vol 25 chapter $5 \mathrm{pp}$ 650-666

[9] Fleiner R, Szasz B and Micsik A 2017 OLOUD - An Ontology for Linked Open University Data, Acta Polytechnica Hungarica vol 14 chapter 4 pp 63-82

[10] Kim H 2012 A Personalized Recommendation Method Using a Tagging Ontology for a Social E-Learning System Proc. of the 3th Intern. Conf., Daegu, Springer Berlin Heidelberg Publ pp 357-366

[11] Sabarish R and Ram Kannan S 2013 Implementation of Ontology in Intelligent E-learning System Development Based on Semantic Web International Journal of Advanced Research in Computer Science and Software Engineering vol 3 chapter 9 pp 474-476

[12] Yusupova N I, Smetanina O N, Klimova A V and Agadullina A I 2019 Information support of academic mobility process using knowledge representation models Modeling, Optimization and Information Technology vol 7 chapter 4 DOI: 10.26102/2310-6018/2019.27.4.017

[13] Chernyakhovskaya L R, Davletbaeva A R and Khabibullina L N 2014 Ontological analysis of the requirements for the quality of e-learning Information technologies in education vol 22 pp 143-149

[14] Smetanina O N, Gayanova M M and Klimova A V 2014 Ontological model for intelligent decision-making support in the formation of educational route taking into account the academic mobility Methodological aspects of artificial intelligence Mechanical engineering pp 38-53

[15] Palagin A V, Kryvyi S L and Petrenko N G 2012 Ontological methods and means of processing subject knowledge: Monograph / Lugansk: publishing house of the VNU. V. Dahl p 324

[16] Volegzhanina I S 2015 Place of ontology researches in the modern education in russia Crimean scientific herald vol 5 pp 26-46

[17] Klimova A V 2014 Algorithmic support for selecting of disciplines in the formation documents within the academic mobility of students Vestnik Ugatu vol 18 chapter 5 pp 177-180 